MATHEMATICS OF COMPUTATION

Volume 71, Number 239, Pages 923-943

S 0025-5718(01)01376-X

Article electronically published on November 20, 2001

\title{
ANALYSIS OF A BILINEAR FINITE ELEMENT FOR SHALLOW SHELLS I: APPROXIMATION OF INEXTENSIONAL DEFORMATIONS
}

\author{
VILLE HAVU AND JUHANI PITKÄRANTA
}

\begin{abstract}
We consider a bilinear reduced-strain finite element formulation for a shallow shell model of Reissner-Naghdi type. The formulation is closely related to the facet models used in engineering practice. We estimate the error of this scheme when approximating an inextensional displacement field. We make the strong assumptions that the domain and the finite element mesh are rectangular and that the boundary conditions are periodic and the mesh uniform in one of the coordinate directions. We prove then that for sufficiently smooth fields, the convergence rate in the energy norm is of optimal order uniformly with respect to the shell thickness. In case of elliptic shell geometry the error bound is furthermore quasioptimal, whereas in parabolic and hyperbolic geometries slightly enhanced smoothness is required, except for the degenerate cases where the characteristic lines are parallel with the mesh lines. The error bound is shown to be sharp.
\end{abstract}

\section{INTRODUCTION}

In this paper, which is the first one in a series, we begin the convergence study of a special bilinear finite element for shells. This element has strong engineering roots, and apparently is among the best bilinear finite element formulations known for shells. The element is aimed to be a general "shell element" in the sense that it should handle all characteristic shell deformation types, such as bending-dominated or membrane-dominated deformations or boundary layers, without further problemspecific tuneups. To what extent this (rather ambitious) goal is truly achievable, is our goal to find out.

By now it is well known that reliable numerical modeling of shells by traditional low-order finite element formulations is not an easy task. The most dramatic failure occurs when approximating nearly inextensional (or bending-dominated) deformations by standard low-order elements. In this case an asymptotic approximation failure, known as shear-membrane locking, occurs at the limit of zero shell thickness. Due to the asymptotic locking effect, standard lowest-order elements cannot work in practice unless the mesh spacing is made much smaller than the shell thicknessa rather heavy requirement. Similar numerical difficulties are expected in general when approximating a deformation state with a significant component of bending energy. In particular, the characteristic shell boundary layers belong to such a

Received by the editor April 12, 2000 and, in revised form, September 28, 2000.

2000 Mathematics Subject Classification. Primary 65N30; Secondary 74K25.

Key words and phrases. Finite elements, locking, shells.

(C)2001 American Mathematical Society 
locking-sensitive category of deformations. For more information on shell deformation states and on the associated finite element locking problems, the reader is referred to [10, 8, 2] and the references therein.

To avoid the locking in parameter dependent problems, the tradition in finite element engineering has been to search for "simple and efficient" low(est) order elements that are based on some nonstandard variational formulation of the problem. For example, one may use mixed or hybrid formulations, or assume purely numerical modifications such as reduced strains, or selective reduced integration and stabilization, within the usual energy formulation. In shell problems, one of the most popular approaches is the so-called facet model based on bilinear elements and crude approximation of shell geometry. In this model, the shell midsurface is approximated elementwise by isoparametric bilinear maps, so that the shell is effectively represented by an assembly of membrane elements in the numerical model [4]. Among the (apparently many) possible technical variations within this approach, we choose to consider in our work a formulation by Bathe et al. [1 named MITC4. It seems likely that certain other well-known formulations, like the QUAD4 of MacNeal 4, hide similar ideas.

Our error analysis of MITC4 is based on the recent result [6] showing that a nearly equivalent bilinear element formulation is achievable from a classical shell model of Reissner-Naghdi type. This alternative approach may be characterized as a reduced-strain formulation where the expressions of both membrane and transverse shear strains are numerically modified, while no approximation of shell geometry is made. This approach, which is more transparent mathematically than the original MITC4 formulation, was in fact already taken in [9] in the context of cylindrical shell geometry. The analysis in [6] assumes cylindrical shell geometry as well, but we may easily extrapolate the strain reduction ideas to more general geometries. It appears that the scheme obtained in this way actually extracts the key ideas of the MITC4 elements (and perhaps other facet elements) that relate to the treatment of locking effects. Numerical experiments also support this conjecture [6]. In what follows, we take the simplified scheme as the target of our error analysis.

Regarding both the shell geometry and the finite element mesh, we make very strong assumptions in this paper and also in the later parts. First, we assume that the curvature parameters can be taken constants (so that the shell can be e.g., shallow); see [8] for the justification of models of this kind. Secondly, we assume that the shell midsurface (or the approximate midsurface; see 8) occupies a rectangular domain in the chosen coordinates and that the finite element mesh on this domain is rectangular. Finally, we assume that the boundary conditions are periodic in one of the coordinate directions, and that the finite element mesh in this direction is actually uniform. Under these hypotheses, we focus on the most fundamental question concerning bilinear shell elements: Does there exist a formulation that is able to effectively capture all characteristic features of shell deformations simultaneously?

From the practical point of view, the most important question left open in our work is, To what extent can the results obtained under the stated extremely favorable conditions possibly extend to more general domains and meshes? Preliminary numerical experiments seem to indicate mesh dependence [5, 6], but more work in this direction is needed. From our error analysis it appears anyhow that the problem on general quadrilateral meshes is similar to that met in [3] in the context of a 
simpler parametric model problem. There it was shown that the convergence of a simple bilinear reduced-strain scheme depends on the performance of an underlying finite difference scheme that approximates a constraint arising asymptotically at a parametric limit. On a rectangular mesh the difference scheme was found well behaving - in fact well known 3] - but on more general meshes the performance remained unresolved. Here we find similarly that the assumed reduced-strain formulation hides a special finite difference scheme for approximating the asymptotic constraints that arise in an inextensional deformation state. This difference scheme we can so far analyze only under the stated specific assumptions.

The assumptions we make are simply to allow sharp error analysis based on the Fourier transform. The same analysis technique would apply to any shell of revolution satisfying the assumed periodicity condition in the angular (principal curvature coordinate) direction. We conjecture that our error analysis could be extended to cover, e.g., the pinched cylinder and the pinched hemisphere problems often used as benchmark tests for shell elements [4. We note that in such benchmark tests, the mesh is typically chosen uniform in the angular direction, just as we are assuming.

In the present Part I, we begin by studying the mentioned constrained approximation problem that arises in an inextensional deformation state. This case is the first and most severe test for any finite element formulation meant to be lockingfree. However, passing the test still does not guarantee equally good performance in other (in fact, more common) deformation states, such as in membrane-dominated states or when approximating boundary layers. Therefore, in the later parts of the work we consider such deformation types as well so as to obtain a complete picture.

Throughout the work we adopt the error analysis philosophy of [3, 1] (see also the references therein) where the finite element error, evaluated in the energy (semi)norm with numerically modified strains, is split into two components called the approximation error and the consistency error. In the present Part I we consider only the approximation error term which is dominant in case of nearly inextensional deformations. The consistency error will be analyzed in the next parts in the context of more general deformation states where this error term can become dominant as well.

The plan of the present paper is as follows. In Section 2 we introduce the shell model and set up the problem to be studied. The nature of inextensional deformations in this problem setup is analyzed. In Section 3 we introduce the reduced-strain bilinear finite element scheme to be studied throughout the work. We state the main result of the present paper concerning the approximation of inextensional deformations (Theorem 3.1) and an extension of this result to cover more general smooth deformations (Theorem 3.2). In the inextensional case the approximation error bound is found to be of optimal order independently of the thickness of the shell. In the case of elliptic shell geometry the bound is optimal even with respect to the regularity required for the displacement field; whereas in the case of parabolic and hyperbolic shell geometries, slightly enhanced regularity is needed as a rule to achieve the optimal rate. For more general than inextensional deformation, the approximation error bound is nonuniform in $t$, being in general the better, the higher the regularity of the field to be approximated. Section 4 is finally devoted to the proof of Theorems 3.1 and 3.2

Below we denote as usual by $C$ a constant that may take a different value in different usage. The constants may depend on the curvature parameters to be introduced in Section 2 but are otherwise independent of the parameters, unless 
indicated explicitly. By $\|\cdot\|_{k}$ and $|\cdot|_{k}$ we denote the Sobolev norm and seminorm (for scalar or vector valued functions) on the assumed rectangular plane domain. Other domains will be indicated by an additional subscript. Further, $\|\cdot\|_{L^{2}}=\|\cdot\|_{0}$ and $\|\cdot\|_{\infty}$ denotes the $L^{\infty}$-norm.

\section{THE SHELl PROBLEM}

We consider a dimensionally reduced shell model for a shell of thickness $t$ arising from linear shell theory with homogeneous and isotropic material. For our model the deformation energy of the shell is given by

$$
\begin{aligned}
\mathcal{F}(u, v, w, \theta, \psi)= & \frac{E t^{3}}{12\left(1-\nu^{2}\right)} \int_{\Omega}\left\{\nu\left(\kappa_{11}+\kappa_{22}\right)^{2}+(1-\nu) \sum_{i, j=1}^{2} \kappa_{i j}^{2}\right\} d x d y \\
& +\frac{\gamma E t}{2(1+\nu)} \int_{\Omega}\left\{\rho_{1}^{2}+\rho_{2}^{2}\right\} d x d y \\
& +\frac{E t}{\left(1-\nu^{2}\right)} \int_{\Omega}\left\{\nu\left(\beta_{11}+\beta_{22}\right)^{2}+(1-\nu) \sum_{i, j=1}^{2} \beta_{i j}^{2}\right\} d x d y .
\end{aligned}
$$

Here $E$ and $\nu$ are the Young modulus and the Poisson ratio of the material, $\gamma$ is the shear (correction) factor and $\beta_{i j}, \rho_{i}$ and $\kappa_{i j}$ are the membrane, transverse shear and bending strains that depend on the displacements $(u, v, w)$ and on the rotations $(\theta, \psi)$ as follows:

$$
\begin{aligned}
\beta_{11} & =\frac{\partial u}{\partial x}+a w, & \kappa_{11} & =\frac{\partial \theta}{\partial x}, \\
\beta_{22} & =\frac{\partial v}{\partial y}+b w, & \kappa_{22} & =\frac{\partial \psi}{\partial y}, \\
\beta_{12} & =\frac{1}{2}\left(\frac{\partial u}{\partial y}+\frac{\partial v}{\partial x}\right)+c w=\beta_{21}, & \kappa_{12} & =\frac{1}{2}\left(\frac{\partial \theta}{\partial y}+\frac{\partial \psi}{\partial x}\right)=\kappa_{21},
\end{aligned}
$$

and

$$
\rho_{1}=\theta-\frac{\partial w}{\partial x}, \quad \rho_{2}=\psi-\frac{\partial w}{\partial y},
$$

where we are assuming that the parameters $a, b$ and $c$, defining the shell geometry, can be taken to be constants. This is a simplification of the classical shell model due to Reissner-Novozhilov-Naghdi (see [8]). If $a b-c^{2}>0$, we call, the shell elliptic, if $a b-c^{2}=0$, the shell is parabolic and if $a b-c^{2}<0$, it is hyperbolic. We assume here that $a^{2}+b^{2}+c^{2}>0$ so that the case of a flat plate/membrane $(a=b=c=0)$ is excluded. We will assume that the computational domain $\Omega$ (the shell midsurface) is of rectangular shape in the assumed coordinate system, so that

$$
\Omega=\{(x, y) \mid 0<x<L, 0<y<H\}
$$

with $C^{-1} \leq \frac{L}{H} \leq C$ for some fixed constant $C$.

For our purposes it is convenient to define the vector field $\underline{u}=(u, v, w, \theta, \psi)$, to scale the energy by a factor $K=\frac{E t^{3}}{6\left(1-\nu^{2}\right)}$, and to express the scaled total energy as

$$
\mathcal{F}(\underline{u})=\frac{1}{2} \mathcal{A}(\underline{u}, \underline{u})-Q(\underline{u}),
$$


where $Q$ is the load potential and the bilinear form $\mathcal{A}$ is further split as

$$
\mathcal{A}(\underline{u}, \underline{v})=\mathcal{A}_{b}(\underline{u}, \underline{v})+\frac{1}{t^{2}} \mathcal{A}_{m}(\underline{u}, \underline{v})
$$

where

$$
\mathcal{A}_{b}(\underline{u}, \underline{v})=\int_{\Omega}\left\{\nu\left(\kappa_{11}+\kappa_{22}\right)(\underline{u})\left(\bar{\kappa}_{11}+\bar{\kappa}_{22}\right)(\underline{v})+(1-\nu) \sum_{i, j=1}^{2} \kappa_{i j}(\underline{u}) \bar{\kappa}_{i j}(\underline{v})\right\} d x d y
$$

and

$$
\begin{array}{r}
\mathcal{A}_{m}(\underline{u}, \underline{v})=6 \gamma(1-\nu) \int_{\Omega}\left\{\rho_{1}(\underline{u}) \bar{\rho}_{1}(\underline{v})+\rho_{2}(\underline{u}) \bar{\rho}_{2}(\underline{v})\right\} d x d y \\
+12 \int_{\Omega}\left\{\nu\left(\beta_{11}+\beta_{22}\right)(\underline{u})\left(\bar{\beta}_{11}+\bar{\beta}_{22}\right)(\underline{v})\right. \\
\left.+(1-\nu) \sum_{i, j=1}^{2} \beta_{i j}(\underline{u}) \bar{\beta}_{i j}(\underline{v})\right\} d x d y
\end{array}
$$

where overbars denote complex conjugation.

We will assume that the boundary conditions at $y=0, H$ are periodic and that no kinematic constraints are imposed at $x=0, L$. The energy space is then

$$
\mathcal{U}=\left\{\underline{u} \in\left[H^{1}(\Omega)\right]^{5} \mid \underline{u}(\cdot, 0)=\underline{u}(\cdot, H)\right\}
$$

and the shell problem can be formulated as, Find $\underline{u} \in \mathcal{U}$ such that

$$
\mathcal{A}(\underline{u}, \underline{v})=Q(\underline{v}) \quad \forall \underline{v} \in \mathcal{U} .
$$

The problem is solvable if $Q$ is a bounded linear functional on $\mathcal{U}$, and if $Q(\underline{u})=0$ for all zero energy modes satisfying $\mathcal{A}(\underline{u}, \underline{u})=0$. Below we do not need to refer to any stability properties of the problem, so we simply assume that a solution to (2.2) (with some regularity properties to be postulated) exists. We then consider the finite element approximation of such a solution $\underline{u}$.

In the present paper we are mainly interested in the approximation of inextensional displacement fields satisfying $\mathcal{A}_{m}(\underline{u}, \underline{u})=0$, i.e.,

$$
\begin{aligned}
\beta_{11}(\underline{u}) & =\beta_{12}(\underline{u})=\beta_{22}(\underline{u})=0, \\
\rho_{1}(\underline{u}) & =\rho_{2}(\underline{u})=0 .
\end{aligned}
$$

The subspace of inextensional displacements will be denoted by $\mathcal{U}_{0}$. We note that unless $Q(\underline{u})=0 \forall \underline{u} \in \mathcal{U}_{0}$, the solution to (2.2) becomes inextensional at the limit when $t \rightarrow 0$ and $Q$ is fixed (see [9, 10]). Here we may resolve the possible inextensional modes by the Fourier expansion

$$
\underline{u}=\sum_{\lambda \in \Lambda} \varphi_{\lambda}(y) \underline{\phi}_{\lambda}(x), \quad \varphi_{\lambda}(y)=e^{i \lambda y}, \quad \Lambda=\left\{\lambda=\frac{2 \pi \nu}{H}, \nu \in \mathbb{Z}\right\},
$$

where, in view of (2.3), $\underline{\phi}_{\lambda}=\left(u_{\lambda}, v_{\lambda}, w_{\lambda}, \theta_{\lambda}, \psi_{\lambda}\right)$ satisfies

$$
\left\{\begin{array}{l}
u_{\lambda}^{\prime}+a w_{\lambda}=0 \\
i \lambda v_{\lambda}+b w_{\lambda}=0 \\
i \lambda u_{\lambda}+v_{\lambda}^{\prime}+2 c w_{\lambda}=0 \\
\theta_{\lambda}-w_{\lambda}^{\prime}=0 \\
\psi_{\lambda}-i \lambda w_{\lambda}=0
\end{array}\right.
$$

Let us characterize the general solution to this system in different geometries. 
First, in the elliptic case where $a b-c^{2}>0$, or in the nondegenerate hyperbolic case where $a b-c^{2}<0$ and $b \neq 0$, the general solution to (2.5) takes the form

$$
\underline{\phi}_{\lambda}(x)=A_{\lambda} \underline{\zeta}_{\lambda, 1}(x)+B_{\lambda} \underline{\zeta}_{\lambda, 2}(x),
$$

where $A_{\lambda}, B_{\lambda}$ are arbitrary complex coefficients and $\underline{\zeta}_{\lambda, k}$ are defined by

$$
\left\{\begin{array}{l}
\underline{\zeta}_{\lambda, 1}(x)=\left(2 c+i \lambda^{-1} \mu_{1} b, b,-i \lambda,-i \lambda \mu_{1}, \lambda^{2}\right) e^{\mu_{1} x} \\
\underline{\zeta}_{\lambda, 2}(x)=\left(2 c+i \lambda^{-1} \mu_{2} b, b,-i \lambda,-i \lambda \mu_{2}, \lambda^{2}\right) e^{\mu_{2}(x-L)} \quad \text { when } \lambda \neq 0,
\end{array}\right.
$$

or

$$
\underline{\zeta}_{\lambda, 1}=(1,0,0,0,0), \quad \underline{\zeta}_{\lambda, 2}=(0,1,0,0,0), \quad \text { when } \lambda=0,
$$

where further $\mu_{k}$ are defined by

$$
\left\{\begin{array}{l}
\mu_{1}=\left(i c-\sqrt{a b-c^{2}}\right) b^{-1} \lambda, \mu_{2}=\left(i c+\sqrt{a b-c^{2}}\right) b^{-1} \lambda, \text { when } b^{-1} \lambda \geq 0, \\
\mu_{1}=\left(i c+\sqrt{a b-c^{2}}\right) b^{-1} \lambda, \mu_{2}=\left(i c-\sqrt{a b-c^{2}}\right) b^{-1} \lambda, \text { when } b^{-1} \lambda<0 .
\end{array}\right.
$$

Here we have chosen $\mu_{1}, \mu_{2}$ so that $\operatorname{Re} \mu_{1}<0$ and $\operatorname{Re} \mu_{2}>0$ in the elliptic case when $\lambda \neq 0$. In the hyperbolic case we obviously have $\operatorname{Re} \mu_{k}=0$. Note also that when $\lambda=0,(2.6)$ and (2.7) actually define a zero energy mode.

Consider next the (hyperbolic) case where $b=0$ and $c \neq 0$. Then if $a \neq 0$ and $\lambda \neq 0$, the general solution to (2.5) is

$$
\underline{\phi}_{\lambda}(x)=A_{\lambda} \underline{\zeta}_{\lambda}(x)
$$

with $A_{\lambda} \in \mathbb{C}$ and

$$
\underline{\zeta}_{\lambda}(x)=\left(2 c, 0,-i \lambda,-i \lambda \mu, \lambda^{2}\right) e^{\mu x}, \mu=a(2 c)^{-1} i \lambda
$$

so the solution space is one-dimensional in this case. When $\lambda=0$ one gets

$$
\underline{\phi}_{0}(x)=\left(a \xi+A, 2 c \xi,-\xi^{\prime},-\xi^{\prime \prime}, 0\right),
$$

where $A \in \mathbb{C}$ and $\xi=\xi(x) \in H^{3}(0, L)$ are arbitrary, so in this case the solution space is infinite-dimensional. Finally, if $a=0$ and $\lambda \neq 0$, one gets again a solution of the form (2.8) with

$$
\underline{\zeta}_{\lambda}(x)=\left(2 c, 0,-i \lambda, 0, \lambda^{2}\right) .
$$

When $a=\lambda=0$, the solution is

$$
\underline{\phi}_{0}=\left(A_{0}, 2 c \xi,-\xi^{\prime},-\xi^{\prime \prime}, 0\right)
$$

with $\xi \in H^{3}(0, L)$ arbitrary.

It remains to consider the parabolic case $a b-c^{2}=0$. Here we find that when $a, b \neq 0$ the general solution is again given by (2.6) where now $\underline{\zeta}_{\lambda, 1}, \underline{\zeta}_{\lambda, 2}$ are given by

$$
\left\{\begin{array}{l}
\underline{\zeta}_{\lambda, 1}(x)=\left(c, b,-i \lambda,-i \lambda \mu, \lambda^{2}\right) e^{\mu x} \\
\underline{\zeta}_{\lambda, 2}(x)=\mu\left(c, b,-i \lambda,-i \lambda \mu, \lambda^{2}\right) x e^{\mu x}+(-c, 0,0,-i \lambda \mu, 0) e^{\mu x}
\end{array}\right.
$$

with $\mu=c b^{-1} i \lambda$. For the case when $a=c=0$ and we have that

$$
\left\{\begin{array}{l}
\underline{\zeta}_{\lambda, 1}(x)=\left(1,-i \lambda b x,-\lambda^{2} x,-\lambda^{2},-i \lambda^{3} x\right) \\
\underline{\zeta}_{\lambda, 2}(x)=\left(0, b,-i \lambda, 0, \lambda^{2}\right)
\end{array}\right.
$$


Finally in the case when $b=c=0$ the only nonzero mode is obtained when $\lambda=0$, this being

$$
\underline{\phi}_{0}(x)=\left(a \xi, A,-\xi^{\prime},-\xi^{\prime \prime}, 0\right)
$$

with $A \in \mathbb{C}$ and $\xi \in H^{3}(0, L)$ arbitrary.

Below we refer to the parabolic and hyperbolic geometries as degenerate cases when the characteristic lines (along which the curvature vanishes) are parallel with the coordinate lines. These are the cases $a=c=0$ or $b=c=0$ (parabolic) and $a=b=0$ (hyperbolic), i.e., the cases where two of the three curvature parameters vanish.

\section{The REDUCED-STRAin FE SCheme}

Given a family of finite element spaces $\mathcal{U}_{h} \subset \mathcal{U}, 0<h<h_{0}$, the usual finite element formulation of problem (2.2) is, Find $\underline{u}_{h} \in \mathcal{U}_{h}$ such that

$$
\mathcal{A}\left(\underline{u}_{h}, \underline{v}\right)=Q(\underline{v}) \quad \forall \underline{v} \in \mathcal{U}_{h} .
$$

This defines $\underline{u}_{h}$ as the best approximation of $\underline{u}$ in the energy norm

$$
\underline{v} \mapsto\|\underline{v}\| \|=\sqrt{\mathcal{A}(\underline{v}, \underline{v})}, \underline{v} \in \mathcal{U} .
$$

Due to the parametric dependence of this norm, the best error bound in case of lowest degree elements and bending-dominated deformation is

$$
|| \underline{u}-\underline{u}_{h} \mid \| \sim \min \left\{1, \frac{h}{t}|\underline{u}|_{2}\right\}
$$

(see [9]). To prevent the error amplification at small $t$, we therefore need to consider some modification of (3.1). A natural approach is to modify the membrane and transverse stresses $\beta_{i j}$ and $\rho_{i}$ substituting these with $\tilde{\beta}_{i j}=R_{h}^{i j} \beta_{i j}$ and $\tilde{\rho}_{i}=R_{h}^{i} \rho_{i}$, where the $R_{h}^{i j}$,s and $R_{h}^{i}$ 's are suitably chosen reduction (projection) operators. If we then define the bilinear form

$$
\begin{array}{r}
\mathcal{A}_{m}^{h}(\underline{u}, \underline{v})=6 \gamma(1-\nu) \int_{\Omega}\left\{\tilde{\rho}_{1}(\underline{u}) \overline{\tilde{\rho}}_{1}(\underline{v})+\tilde{\rho}_{2}(\underline{u}) \overline{\tilde{\rho}}_{2}(\underline{v})\right\} d x d y \\
+12 \int_{\Omega}\left\{\nu\left(\tilde{\beta}_{11}+\tilde{\beta}_{22}\right)(\underline{u})\left(\overline{\tilde{\beta}}_{11}+\overline{\tilde{\beta}}_{22}\right)(\underline{v})\right. \\
\left.+(1-\nu) \sum_{i, j=1}^{2} \tilde{\beta}_{i j}(\underline{u}) \overline{\tilde{\beta}}_{i j}(\underline{v})\right\} d x d y
\end{array}
$$

our modified FE formulation assumes the form, Find $\underline{u}_{h} \in \mathcal{U}_{h}$ such that

$$
\mathcal{A}_{h}\left(\underline{u}_{h}, \underline{v}\right) \doteqdot \mathcal{A}_{b}\left(\underline{u}_{h}, \underline{v}\right)+\frac{1}{t^{2}} \mathcal{A}_{m}^{h}\left(\underline{u}_{h}, \underline{v}\right)=Q(\underline{v}) \quad \forall \underline{v} \in \mathcal{U}_{h} .
$$

Since $\tilde{\beta}_{i j}, \tilde{\rho}_{i}$ are related to $\beta_{i j}, \rho_{i}$ via projection operators, it follows that $\mathcal{A}_{m}^{h}(\underline{u}, \underline{u})=$ 0 for all $\underline{u} \in \mathcal{U}_{0}$. Therefore, if the exact solution to (2.2) happens to be inextensional, one has

$$
Q(\underline{v})=\mathcal{A}(\underline{u}, \underline{v})=\mathcal{A}_{b}(\underline{u}, \underline{v})=\mathcal{A}_{h}(\underline{u}, \underline{v}), \underline{v} \in \mathcal{U} .
$$

So in that case (3.3) is equivalent to

$$
\mathcal{A}_{h}\left(\underline{u}-\underline{u}_{h}, \underline{v}\right)=0 \quad \forall \underline{v} \in \mathcal{U}_{h} .
$$




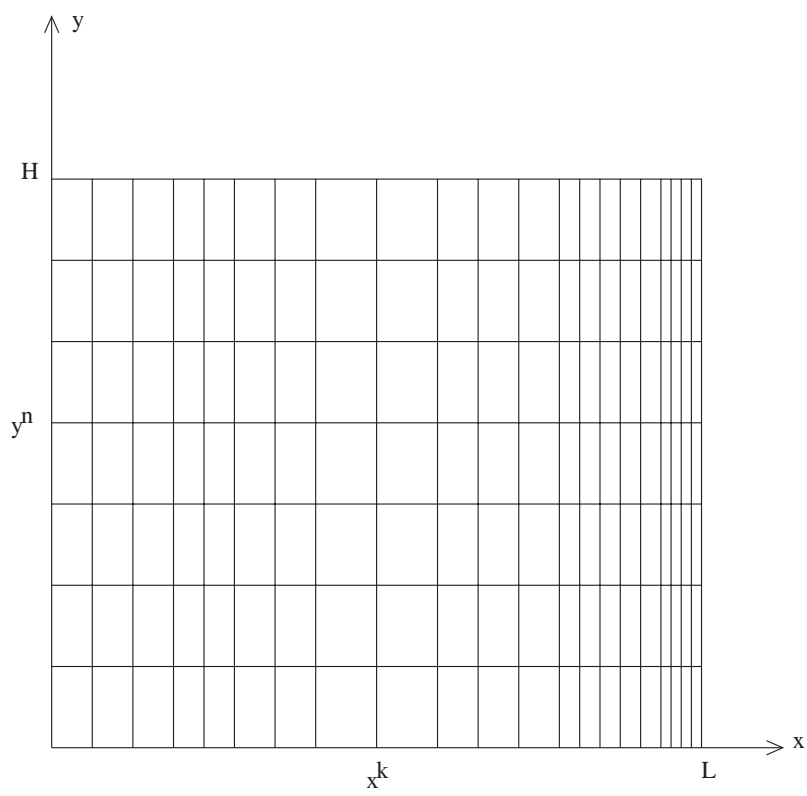

Figure 1. A typical mesh considered; the mesh spacing is constant in the $y$-direction but may vary in the $x$-direction.

This defines $\underline{u}_{h}$ as the best approximation to $\underline{u}$ in the modified energy seminorm $\underline{v} \mapsto\|\underline{v}\|_{h}=\sqrt{\mathcal{A}_{h}(\underline{v}, \underline{v})}$, so that we are led to the problem of finding the best approximation error

$$
e_{a}(\underline{u})=\min _{\underline{v} \in \mathcal{U}_{h}}\|\underline{u}-\underline{v} \mid\|_{h}
$$

We note that, to obtain a bound for $e_{a}$ that is uniform in $t$, we need to look for approximations in the subspace $\mathcal{U}_{0, h}=\left\{\underline{v} \in \mathcal{U}_{h} \mid \mathcal{A}_{m}^{h}(\underline{v}, \underline{v})=0\right\}$. Note as well that if no strain reductions were made, we would have $\mathcal{U}_{0, h}=\mathcal{U}_{0} \cap \mathcal{U}_{h}$, which typically is a trivial space [9. Hence the idea of the strain reduction is simply to make $\mathcal{U}_{0, h}$ large enough so that inextensional deformations can be approximated well in $\mathcal{U}_{0, h}$.

The modification $\mathcal{A} \hookrightarrow \mathcal{A}_{h}$ causes in general a secondary error term, the consistency error defined by $[\underline{3}$

$$
e_{c}(\underline{u})=\sup _{\underline{v} \in \mathcal{U}_{h},\|\underline{v}\| \|_{h} \neq 0} \frac{\left(\mathcal{A}-\mathcal{A}_{h}\right)(\underline{u}, \underline{v})}{\|\underline{v}\| \|_{h}} .
$$

This vanishes when $\underline{u} \in \mathcal{U}_{0}$, but in other deformation states the consistency error term can be significant or even dominant. We will analyze this error in Part II of the paper where more general deformation states will be considered. Here we focus on bounding the best approximation error (3.4).

In the finite element scheme to be studied, we assume that $\Omega$ is subdivided by a rectangular mesh with maximal side length $=h$. We denote the nodal points of the mesh by $\left(x^{k}, y^{n}\right), k=0 \ldots N_{x}, n=0 \ldots N_{y}$, and assume that the mesh is uniform in the $y$-direction so that $y^{n+1}-y^{n}=H / N_{y}=h_{y}$ for all $n$ (see Figure 1). Note that at this point we make no assumption on the ratios $\left(x^{k+1}-x^{k}\right) / h_{y}=h_{x}^{k} / h_{y}$. We write further $h_{x}=\max _{k} h_{x}^{k}$. On this mesh we assume continuous piecewise bilinear 
representation of each component of the displacement field, so that $\mathcal{U}_{h}=\left[\mathcal{V}_{h}\right]^{5}$, where $\mathcal{V}_{h}$ is the scalar bilinear FE space with periodic boundary conditions at $y=0, H$.

In the assumed numerical setup, we define the reduced membrane and shear strains in (3.2) as

$$
\begin{aligned}
& \tilde{\beta}_{11}=\Pi_{h}^{x} \beta_{11}, \\
& \tilde{\beta}_{22}=\Pi_{h}^{y} \beta_{22}, \\
& \tilde{\beta}_{12}=\Pi_{h}^{x y} \beta_{12}=\tilde{\beta}_{21},
\end{aligned}
$$

and

$$
\begin{aligned}
& \tilde{\rho}_{1}=\Pi_{h}^{x} \rho_{1}, \\
& \tilde{\rho}_{2}=\Pi_{h}^{y} \rho_{2},
\end{aligned}
$$

where $\Pi_{h}^{x} x, \Pi_{h}^{y}$ and $\Pi_{h}^{x y}$ are projection operators defined elementwise as projectors onto the (global) spaces $\mathcal{W}_{h}^{x}, \mathcal{W}_{h}^{y}$, and $\mathcal{W}_{h}^{x y}$, respectively, where $\mathcal{W}_{h}^{x}$ consists of functions that are constant in $x$ and linear with respect to $y$ on each element, so that $\partial u / \partial x \in \mathcal{W}_{h}^{x}$ when $u \in \mathcal{V}_{h}, \mathcal{W}_{h}^{y}$ is defined analogously so that $\partial v / \partial y \in \mathcal{W}_{h}^{y}$ when $v \in \mathcal{V}_{h}$, and $\mathcal{W}_{h}^{x y}$ consists of functions that are constant on each element. We consider these as subspaces of $L^{2}(\Omega)$, so we impose a priori no continuity conditions along the interelement boundaries. Operator $\Pi_{h}^{x y}$ in (3.5) is defined simply as the $L^{2}$ projection, i.e., $\Pi_{h}^{x y}$ is the elementwise averaging operator. When defining $\Pi_{h}^{x}$, $\Pi_{h}^{y}$ we consider two options which lead to the same finite element scheme but differ slightly in the error analysis based on (3.4).

$(\mathrm{O} 1) \Pi_{h}^{x}: L^{2}(\Omega) \rightarrow \mathcal{W}_{h}^{x}, \Pi_{h}^{y}: L^{2}(\Omega) \rightarrow \mathcal{W}_{h}^{y}$ are defined as $L^{2}$-projections.

(O2) $\Pi_{h}^{x}, \Pi_{h}^{y}$ are defined for sufficiently regular $\phi$ so that for each horizontal side $S_{x}$ and for each vertical side $S_{y}$ of the rectangular mesh

$$
\begin{aligned}
\int_{S_{x}} \Pi_{h}^{x} \phi(x, y) d x & =\int_{S_{x}} \phi(x, y) d x, \\
\int_{S_{y}} \Pi_{h}^{y} \phi(x, y) d y & =\int_{S_{y}} \phi(x, y) d y .
\end{aligned}
$$

Remark 3.1. Since the strain reductions by $\Pi_{h}^{x}, \Pi_{h}^{y}$ do not affect the derivative terms of $\beta_{i i}$ and $\rho_{i}$ in (3.5)-(3.6) when $u, v, w \in \mathcal{V}_{h}$, we could obviously write $\tilde{\beta}_{11}=\partial u / \partial x+\Pi_{h}^{x} a w$, etc., in the finite element model. In the FE model we could further interpret the reductions equivalently as interpolation operators, such that $\Pi_{h}^{x}$ interpolates at the midpoints $\left(x^{k+1 / 2}, y^{n}\right)$ of the horizontal sides, $\Pi_{h}^{y}$ interpolates at the midpoints $\left(x^{k}, y^{n+1 / 2}\right)$ of the vertical sides, and $\Pi_{h}^{x y}$ interpolates at the centers $\left(x^{k+1 / 2}, y^{n+1 / 2}\right)$ of the rectangles $K_{k n}$. Although such interpretations do not affect the FE algorithm, they do have an effect on the error indicator (3.4) if assumed beyond $\mathcal{U}_{h}$. Also the two options (O1), (O2) are different in this sense. Option (O1) is natural in the sense that the modified strains are then defined in the entire energy space, and one has $\left\|\underline{v}\left|\left\|_{h} \leq C \mid\right\| \underline{v}\|\|, \underline{v} \in \mathcal{U}\left(\right.\right.\right.$ with $C=\left(1-\nu^{2}\right)^{-1 / 2}$ as the best constant). The advantage of (O2) is that this definition enforces the continuity of $\tilde{\beta}_{11}$ and $\tilde{\rho}_{1}$ with respect to $y$ and the continuity of $\tilde{\beta}_{22}$ and $\tilde{\rho}_{2}$ with respect to $x$. We need this property in Theorem 3.2 below.

Remark 3.2. The connection of the chosen reduced-strain algorithm and the facet FEM models is discussed in [6] in the context of cylindrical shell geometry. In 


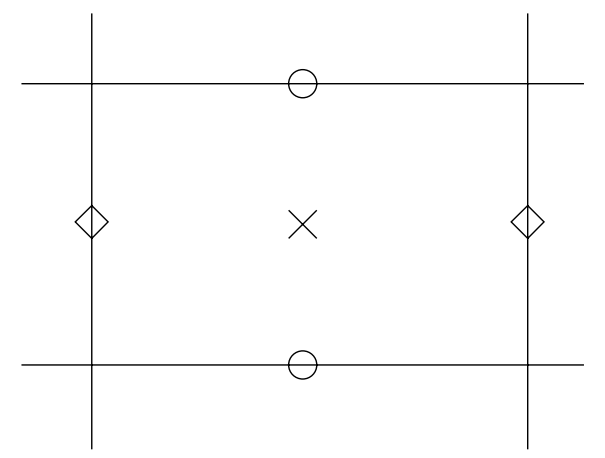

FIGURE 2. Finite difference approximation of the shear and membrane constraints in the reduced-strain $\mathrm{FE}$ formulation: $\rho_{1}=$ $\beta_{11}=0$ at $(\bigcirc), \rho_{2}=\beta_{22}=0$ at $(\diamond)$ and $\beta_{12}=0$ at $(\times)$.

(3.5) - (3.6) we have made an extrapolation from this study (cf. also [9]). We note that the facet formulation actually implies a slightly different definition of $\tilde{\beta}_{12}$, but this reduces essentially to the chosen one when approximating an inextensional deformation [6].

When approximating an inextensional field $\underline{u} \in \mathcal{U}_{0}$, we can bound the best approximation error (3.4) uniformly in $t$ from

$$
e_{a}(\underline{u}) \leq \tilde{e}_{a}(\underline{u})=\min _{\underline{v} \in \mathcal{U}_{0, h}}\|\underline{u}-\underline{v}\|_{h}=\min _{\underline{v} \in \mathcal{U}_{0, h}} \sqrt{\mathcal{A}_{b}(\underline{u}-\underline{v}, \underline{u}-\underline{v})} .
$$

Obviously this also gives the best bound that is uniform in $t$. When $\underline{u} \in \mathcal{U}_{h}$, the reduced constraints $\tilde{\beta}_{i j}(\underline{u})=\tilde{\rho}_{i}(\underline{u})=0, i, j=1,2$ are imposed equivalently by setting $\rho_{1}=\beta_{11}=0$ at the midpoint of each horizontal side of the mesh, $\rho_{2}=\beta_{22}=0$ at the midpoint of each vertical side, and $\beta_{12}=0$ at each element center (see Remark [3.1), so the reduced constraints are effectively finite difference approximations of the original ones (see Figure 2).

Our first main result states an estimate for $\tilde{e}_{a}$ in (3.7) when $\underline{u} \in \mathcal{U}_{0}$.

Theorem 3.1. Let $\mathcal{U}_{0, h}=\left\{\underline{u} \in \mathcal{U}_{h} \mid \mathcal{A}_{m}^{h}(\underline{u}, \underline{u})=0\right\}$, where $\mathcal{U}_{h}$ is the bilinear finite element space and $\mathcal{A}_{m}^{h}$ is defined by (3.2) and (3.5) -3.6) where $\Pi_{h}^{x y}$ is the elementwise averaging operator and $\Pi_{h}^{x}, \Pi_{h}^{y}$ are defined according to either option (O1) or $(\mathrm{O} 2)$. Then, if $\underline{u} \in \mathcal{U}_{0}$, the constrained approximation error (3.7) is bounded as

$$
\tilde{e}_{a}(\underline{u}) \leq C_{1} h|\underline{u}|_{2}+C_{2} h^{\frac{2}{3}(s-1)}|\underline{u}|_{s}, \quad 2 \leq s \leq 3,
$$

where $C_{2}=0$ in the elliptic case and also in the degenerate parabolic or hyperbolic cases.

We note that when bounding $e_{a}$ according to (3.7), the result of Theorem 3.1 is easily extended to cover bending-dominated (or nearly inextensional) fields of the form

$$
\underline{u}=\underline{u}^{0}+t \underline{u}^{1},
$$

where $\underline{u}^{0} \in \mathcal{U}_{0}$ and $\underline{u}^{1} \in \mathcal{U}$ is such that $\left|\underline{u}^{1}\right|_{2}$ is bounded uniformly in $t$. Indeed, the factor $t$ in front of $\underline{u}^{1}$ in (3.8) cancels the parametric error amplification, so that the standard interpolant $t \underline{\tilde{u}} \in \mathcal{U}_{h}$ approximates this term sufficiently accurately. 
For membrane-dominated deformations, or for deformations containing strong layers, the expansion (3.8) is not realistic unless $\left|\underline{u}^{1}\right|_{2}$ is allowed to grow as $t \rightarrow 0$. For such more general deformations we can anyhow extend the result of Theorem 3.1 to a nonuniform bound with respect to $t$, provided $\underline{u}$ is extremely smooth. Since we need also to strengthen our assumptions on the mesh here, let us define the following weak uniformity conditions:

(A) $h / h_{x}^{k} \leq C \quad \forall k$

(B) $\sum_{k=1}^{N_{x}-1}\left|h_{x}^{k}-h_{x}^{k-1}\right| \leq C h$.

Theorem 3.2. Let $\underline{u} \in\left[H^{s}(\Omega)\right]^{5}, s \geq 6$, assume that the mesh satisfies conditions (A), (B), and assume that $\mathcal{A}_{m}^{h}$ in (3.3) is defined as in Theorem [3.1, with $\Pi_{h}^{x}, \Pi_{h}^{y}$ now defined according to option (O2). Then the best approximation error 3.4 is bounded for each $t>0$ as

$$
e_{a}(\underline{u}) \leq C\|\underline{u}\|_{6} h+C\|\underline{u}\|_{s} t^{-1} h^{s-2} .
$$

Remark 3.3. In the case of a smooth membrane-dominated deformation such that the second term in (2.1) is dominating, it would be more natural to scale (2.1) by factor $t^{2}$ and thus the energy norm by factor $t$. In such a weaker norm one would obtain by standard interpolation error analysis the quasioptimal bound $e_{a}(\underline{u}) \leq$ $C|\underline{u}|_{2} h$ (cf. also [3, 7]). The result of Theorem [3.2, which states an error bound in a rather strong norm, is based on nonstandard interpolation.

\section{Proof of Theorems 3.1 and 3.2}

The main idea of the proof is to use the Fourier representation (2.4) (for $\underline{u} \in \mathcal{U}_{0}$ or $\underline{u} \in \mathcal{U}$ ) and approximate then $\underline{u}$ by

$$
\underline{\tilde{u}}=\sum_{\lambda \in \Lambda:|\lambda| \leq \lambda_{0}} \tilde{\varphi}_{\lambda}(y) \tilde{\tilde{\phi}}_{\lambda}(x),
$$

where $\lambda_{0}=\lambda_{0}(h)$ is a truncation frequency to be chosen, $\tilde{\varphi}_{\lambda}$ is the piecewise linear interpolant of $\varphi_{\lambda}$, and $\underline{\phi}_{\lambda}$ is a special approximation of $\underline{\phi}_{\lambda}$ to be found.

Lemma 4.1. Assume that the geometric parameters $a, b$ and $c$ satisfy one of the following:

$$
\left\{\begin{array}{l}
b \neq 0, a \neq 0, c \in \mathbb{R} \\
b \neq 0, c \neq 0, a \in \mathbb{R} \\
b \neq 0, a=0, c=0 .
\end{array}\right.
$$

Then for every $\underline{\vartheta}_{\lambda, j}=\varphi_{\lambda}(y) \underline{\zeta}_{\lambda, j}(x), j=1,2$, such that $|\lambda| h_{y} \leq|\lambda| h \leq \hat{c}<\pi, \hat{c}$ small enough, there exists $\underline{\tilde{\vartheta}}_{\lambda, j}=\tilde{\varphi}_{\lambda}(y) \underline{\tilde{\zeta}}_{\lambda, j}(x) \in \mathcal{U}_{0, h}$ such that

$$
\left\|\underline{\vartheta}_{\lambda, j}-\underline{\tilde{\vartheta}}_{\lambda, j}\left|\|_{h} \leq\left(C_{1} h+C_{2} \lambda^{2} h^{2}\right) \underline{\vartheta}_{\lambda, j}\right|_{2}\right.
$$

with $C_{2}=0$ in the elliptic and in the degenerate parabolic case $a=c=0$.

Proof. Consider first the cases where $b \neq 0$ and either $a \neq 0$ or $c \neq 0$. Without loss of generality we restrict to the case $j=1$ and write $\underline{\zeta}_{\lambda, 1}=\underline{\zeta}_{\lambda}=\left(u_{\lambda}, v_{\lambda}, w_{\lambda}, \theta_{\lambda}, \psi_{\lambda}\right)$ and similarly $\underline{\tilde{\zeta}}_{\lambda, 1}=\underline{\tilde{\zeta}}_{\lambda}=\left(\tilde{u}_{\lambda}, \tilde{v}_{\lambda}, \tilde{w}_{\lambda}, \tilde{\theta}_{\lambda}, \tilde{\psi}_{\lambda}\right)$. Since we want $\underline{\tilde{\vartheta}}_{\lambda} \in \mathcal{U}_{0, h}$, one 
must have $\tilde{\beta}_{i j}\left(\underline{\tilde{\vartheta}}_{\lambda}\right)=\tilde{\rho}_{i}\left(\underline{\tilde{\vartheta}}_{\lambda}\right)=0$. This is equivalent to requiring the nodal values $\underline{\tilde{\vartheta}}_{\lambda}\left(x^{k}, y^{n}\right)=\underline{\tilde{\vartheta}}_{k}^{n}=\left(u_{k}^{n}, v_{k}^{n}, w_{k}^{n}, \theta_{k}^{n}, \psi_{k}^{n}\right)$ to satisfy

$$
\begin{cases}u_{k+1}^{n}-u_{k}^{n}+\frac{a}{2} h_{x}^{k}\left(w_{k}^{n}+w_{k+1}^{n}\right) & =0, \\ v_{k}^{n+1}-v_{k}^{n}+\frac{b}{2} h_{y}\left(w_{k}^{n}+w_{k}^{n+1}\right) & =0, \\ \frac{1}{2 h_{y}}\left(u_{k+1}^{n+1}-u_{k+1}^{n}+u_{k}^{n+1}-u_{k}^{n}\right), & \\ \quad+\frac{1}{2 h_{x}^{k}}\left(v_{k+1}^{n+1}-v_{k}^{n+1}+v_{k+1}^{n}-v_{k}^{n}\right) & \\ \quad++\frac{c}{2}\left(w_{k+1}^{n+1}+w_{k}^{n+1}+w_{k+1}^{n}+w_{k}^{n}\right) & =0, \\ \frac{h_{x}^{k}}{2}\left(\theta_{k}^{n}+\theta_{k+1}^{n}\right)-\left(w_{k+1}^{n}-w_{k}^{n}\right) & =0, \\ \frac{h_{y}}{2}\left(\psi_{k}^{n}+\psi_{k}^{n+1}\right)-\left(w_{k}^{n+1}-w_{n}^{k}\right) & =0 .\end{cases}
$$

Inspired by the form of $\underline{\vartheta}_{\lambda}$, we seek a solution to these equations in the form $\underline{\tilde{\vartheta}}_{k}^{n}=e^{i \lambda n h_{y}}\left(U^{k}, V^{k}, W^{k}, \Theta^{k}, \Psi^{k}\right)$. Substituting these expressions and simplifying, we get from the second and from the last two equations in (4.1) that

$$
\left\{\begin{array}{l}
W^{k}=\frac{-2 i}{b h_{y}} \tan \left(\frac{1}{2} \lambda h_{y}\right) V^{k} \\
\Psi^{k}=\frac{2 i}{h_{y}} \tan \left(\frac{1}{2} \lambda h_{y}\right) W^{k} \\
\Theta^{k+1}+\Theta^{k}=\frac{2}{h_{x}^{k}}\left(W^{k+1}-W^{k}\right)
\end{array}\right.
$$

and from the first three equations in (4.1) that

$$
\left(\begin{array}{l}
V \\
U
\end{array}\right)_{k+1}-\left(\begin{array}{l}
V \\
U
\end{array}\right)_{k}=\frac{1}{2} \tau_{k} M\left[\left(\begin{array}{l}
V \\
U
\end{array}\right)_{k+1}+\left(\begin{array}{l}
V \\
U
\end{array}\right)_{k}\right],
$$

where $\tau_{k}=2 \frac{h_{x}^{k}}{h_{y}} \tan \left(\frac{1}{2} \lambda h_{y}\right)$ and

$$
M=i\left(\begin{array}{cc}
\frac{2 c}{b} & -1 \\
\frac{a}{b} & 0
\end{array}\right) .
$$

Since $u_{\lambda}$ and $v_{\lambda}$ satisfy by (2.5) a system

$$
\left(\begin{array}{l}
v_{\lambda} \\
u_{\lambda}
\end{array}\right)^{\prime}=\lambda M\left(\begin{array}{l}
v_{\lambda} \\
u_{\lambda}
\end{array}\right)
$$

we see that (4.3) is a finite difference approximation to (4.4) that closely resembles the Trapezoidal rule. The first step is now to perform the error analysis of (4.3).

We consider the approximation of a solution to (4.4) of the form $\left(u_{\lambda}, v_{\lambda}\right) \sim e^{-\mu x}$, where $\mu=\left(\alpha_{1}+i \alpha_{2}\right) \lambda=\alpha \lambda$ with $\alpha_{1} \lambda>0$ in the elliptic case when $\lambda \neq 0$ and $\alpha_{1}=0$ in the parabolic and hyperbolic case. Without loss of generality we may assume that $|\lambda| h_{y} \leq|\lambda| h \leq \hat{c}<\pi$. (This sets an upper bound of order $O\left(h^{-1}\right)$ for the truncation frequency $\lambda_{0}$.) Then

$$
\begin{aligned}
\frac{1-\frac{1}{2} \alpha \tau_{k}}{1+\frac{1}{2} \alpha \tau_{k}} & =e^{-\mu h_{x}^{k}}+O\left(|\lambda|^{3}\left(h_{x}^{k}\right)^{3}+|\lambda|^{3} h_{x}^{k}\left(h_{y}\right)^{2}\right), \\
\left|\frac{1-\frac{1}{2} \alpha \tau_{k}}{1+\frac{1}{2} \alpha \tau_{k}}\right| & \leq e^{-\beta|\lambda| h_{x}^{k}},
\end{aligned}
$$

where $\alpha_{1}>\beta>0$ in the elliptic case and $\beta=0$ in the parabolic and hyperbolic case. Following the standard error analysis techniques for A-stable difference schemes (for 
the reader's convenience we present the reasoning in Appendix $\mathrm{A}$, we then see that with the initial conditions $\tilde{u}_{\lambda}(0)=u_{\lambda}(0), \tilde{v}_{\lambda}(0)=v_{\lambda}(0)$,

$$
\begin{aligned}
\left|v_{\lambda}\left(x^{k}\right)-\tilde{v}_{\lambda}\left(x^{k}\right)\right| & \leq C \int_{0}^{x^{k}} e^{-\beta|\lambda|\left(x^{k}-t\right)}|\lambda|^{3}\left(h_{x}^{2}+h_{y}^{2}\right) e^{-\alpha_{1}|\lambda| t} d t \\
& \leq C h^{2}|\lambda|^{3-m} e^{-\beta|\lambda| x^{k}}
\end{aligned}
$$

where

$$
m= \begin{cases}1 & \text { in the elliptic case } \\ 0 & \text { in the parabolic and hyperbolic case }\end{cases}
$$

Comparing (4.3), (4.4) and using (4.5) we also see that

$$
\left|v_{\lambda}^{\prime}\left(x^{k+1 / 2}\right)-\tilde{v}_{\lambda}^{\prime}\left(x^{k+1 / 2}\right)\right| \leq C h^{2}|\lambda|^{4-m} e^{-\beta|\lambda| x^{k}} .
$$

Furthermore, from the first two equalities in (4.2) and from their continuous counterparts we conclude easily that (4.5) and (4.6) imply

$$
\begin{aligned}
\left|w_{\lambda}\left(x^{k}\right)-\tilde{w}_{\lambda}\left(x^{k}\right)\right| & \leq C h^{2}|\lambda|^{4-m} e^{-\beta|\lambda| x^{k}}, \\
\left|\psi_{\lambda}\left(x^{k}\right)-\tilde{\psi}_{\lambda}\left(x^{k}\right)\right| & \leq C h^{2}|\lambda|^{5-m} e^{-\beta|\lambda| x^{k}}, \\
\left|\psi_{\lambda}^{\prime}\left(x^{k+1 / 2}\right)-\tilde{\psi}_{\lambda}^{\prime}\left(x^{k+1 / 2}\right)\right| & \leq C h^{2}|\lambda|^{6-m} e^{-\beta|\lambda| x^{k}} .
\end{aligned}
$$

Upon combining the last two bounds in (4.7) with standard interpolation error bounds and using finally $|\lambda|^{4-\frac{1}{2} m} \leq C\left|\varphi_{\lambda}(y) \psi_{\lambda}(x)\right|_{2}$, we obtain the following $L^{2}$ bounds:

$$
\begin{aligned}
& \left\|\psi_{\lambda}-\tilde{\psi}_{\lambda}\right\|_{L^{2}(0, L)} \leq C h^{2}|\lambda|^{1-m}\left|\varphi_{\lambda} \psi_{\lambda}\right|_{2}, \\
& \left\|\psi_{\lambda}^{\prime}-\tilde{\psi}_{\lambda}^{\prime}\right\|_{L^{2}(0, L)} \leq\left(C h+C h^{2}|\lambda|^{2-m}\right)\left|\varphi_{\lambda} \psi_{\lambda}\right|_{2} .
\end{aligned}
$$

Using (4.8) together with $\left\|\varphi_{\lambda}-\tilde{\varphi}_{\lambda}\right\|_{L^{2}(0, H)} \leq C h^{2} \lambda^{2},\left\|\varphi_{\lambda}^{\prime}-\tilde{\varphi}_{\lambda}^{\prime}\right\|_{L^{2}(0, H)} \leq C h \lambda^{2}$, $\lambda^{2}\left\|\psi_{\lambda}\right\|_{L^{2}(0, L)} \leq C\left|\varphi_{\lambda} \psi_{\lambda}\right|_{2},|\lambda||| \psi_{\lambda}^{\prime} \|_{L^{2}(0, L)} \leq C\left|\varphi_{\lambda} \psi_{\lambda}\right|_{2}$, and recalling also that $|\lambda| h \leq \hat{c}$, we can bound the second term on the right hand side of

$$
\begin{aligned}
\left.\left\|\underline{\vartheta}_{\lambda}-\underline{\tilde{\vartheta}}_{\lambda}\right\|\right|_{h} & =\mathcal{A}_{b}\left(\underline{\vartheta}_{\lambda}-\underline{\tilde{\vartheta}}_{\lambda}, \underline{\vartheta}_{\lambda}-\underline{\tilde{\vartheta}}_{\lambda}\right)^{1 / 2} \\
& \leq C\left|\varphi_{\lambda} \theta_{\lambda}-\tilde{\varphi}_{\lambda} \tilde{\theta}_{\lambda}\right|_{1}+C\left|\varphi_{\lambda} \psi_{\lambda}-\tilde{\varphi}_{\lambda} \tilde{\psi}_{\lambda}\right|_{1}
\end{aligned}
$$

from

$$
\begin{aligned}
\left|\varphi_{\lambda}(y) \psi_{\lambda}(x)-\tilde{\varphi}_{\lambda}(y) \tilde{\psi}_{\lambda}(x)\right|_{1} \leq & \left|\left(\varphi_{\lambda}(y)-\tilde{\varphi}_{\lambda}(y)\right) \psi_{\lambda}(x)\right|_{1} \\
& +\left|\tilde{\varphi}_{\lambda}(y)\left(\psi_{\lambda}(x)-\tilde{\psi}_{\lambda}(x)\right)\right|_{1}
\end{aligned}
$$

as

$$
\left|\varphi_{\lambda} \psi_{\lambda}-\tilde{\varphi}_{\lambda} \tilde{\psi}_{\lambda}\right|_{1} \leq\left(C_{1} h+C_{2} h^{2} \lambda^{2}\right)\left|\varphi_{\lambda} \psi_{\lambda}\right|_{2},
$$

where $C_{2}=0$ in the elliptic case.

For the second rotational component $\varphi_{\lambda} \theta_{\lambda}$ the estimation can be done similarly up to the point where a bound for $\delta \theta_{\lambda}^{k}=\theta_{\lambda}\left(x^{k}\right)-\tilde{\theta}_{\lambda}\left(x^{k}\right)$ is needed. Since by (4.2) we have that

$$
\frac{1}{2}\left(\tilde{\theta}_{\lambda}\left(x^{k+1}\right)+\tilde{\theta}_{\lambda}\left(x^{k}\right)\right)=\tilde{w}_{\lambda}^{\prime}\left(x^{k+1 / 2}\right)
$$


and clearly

$$
\frac{1}{2}\left(\theta_{\lambda}\left(x^{k+1}\right)+\theta_{\lambda}\left(x^{k}\right)\right)=\frac{1}{2}\left(w_{\lambda}^{\prime}\left(x^{k+1}\right)+w_{\lambda}^{\prime}\left(x^{k}\right)\right),
$$

it follows that

$$
\frac{1}{2}\left(\delta \theta_{\lambda}^{k+1}+\delta \theta_{\lambda}^{k}\right)=\frac{1}{2}\left(w_{\lambda}^{\prime}\left(x^{k+1}\right)+w_{\lambda}^{\prime}\left(x^{k}\right)\right)-\tilde{w}_{\lambda}^{\prime}\left(x^{k+1 / 2}\right)=\omega_{k},
$$

hence

$$
\delta \theta_{\lambda}^{k}=(-1)^{k} \delta \theta_{\lambda}^{0}+2 \sum_{j=1}^{k}(-1)^{j+1} \omega_{k-j} .
$$

In order to bound the right hand side of (4.10), recall first that by (4.3)

$$
\left(\begin{array}{c}
\tilde{v}_{\lambda} \\
\tilde{u}_{\lambda}
\end{array}\right)^{\prime}\left(x^{k+1 / 2}\right)=\frac{1}{h_{y}} \tan \left(\frac{1}{2} \lambda h_{y}\right) M\left[\left(\begin{array}{c}
\tilde{v}_{\lambda} \\
\tilde{u}_{\lambda}
\end{array}\right)\left(x^{k}\right)+\left(\begin{array}{c}
\tilde{v}_{\lambda} \\
\tilde{u}_{\lambda}
\end{array}\right)\left(x^{k+1}\right)\right] .
$$

Since also

$$
\tilde{w}_{\lambda}^{\prime}\left(x^{k+1 / 2}\right)=-\frac{2 i}{b h_{y}} \tan \left(\frac{1}{2} \lambda h_{y}\right) \tilde{v}_{\lambda}^{\prime}\left(x^{k+1 / 2}\right)
$$

and

$$
w_{\lambda}^{\prime}\left(x^{k}\right)=-\frac{i \lambda}{b} v_{\lambda}^{\prime}\left(x^{k}\right)
$$

we see that

$$
\begin{aligned}
\omega_{k}= & \frac{2}{b}\left[\left(\frac{\lambda^{2}}{4}-\left(\frac{1}{h_{y}} \tan \left(\frac{1}{2} \lambda h_{y}\right)\right)^{2}\right)\left(\frac{2 c}{b}\left(v_{\lambda}\left(x^{k+1}\right)+v_{\lambda}\left(x^{k}\right)\right)-\left(u_{\lambda}\left(x^{k+1}\right)+u_{\lambda}\left(x^{k}\right)\right)\right)\right. \\
& \left.\left.+\left(\frac{1}{h_{y}} \tan \left(\frac{1}{2} \lambda h_{y}\right)\right)^{2}\right)\left(\frac{2 c}{b}\left(\delta v_{\lambda}^{k+1}+\delta v_{\lambda}^{k}\right)-\left(\delta u_{\lambda}^{k+1}+\delta u_{\lambda}^{k}\right)\right)\right] \\
= & \Delta_{k+1}+\Delta_{k}
\end{aligned}
$$

where $\delta v_{\lambda}^{k}=v_{\lambda}\left(x^{k}\right)-\tilde{v}_{\lambda}\left(x^{k}\right), \delta u_{\lambda}^{k}=u_{\lambda}\left(x^{k}\right)-\tilde{u}_{\lambda}\left(x^{k}\right)$. By (4.11) the telescoping sum (4.10) reduces to $\delta \theta_{\lambda}^{k}=\Delta_{k}+(-1)^{k+1}\left(2 \Delta_{0}-\delta \theta_{\lambda}^{0}\right)$, so with the initial condition $\delta \theta_{\lambda}^{0}=2 \Delta_{0}$ we get

$$
\left|\theta_{\lambda}\left(x^{k}\right)-\tilde{\theta}_{\lambda}\left(x^{k}\right)\right|=2\left|\Delta_{k}\right| \leq C h^{2}|\lambda|^{5-m} e^{-\beta|\lambda| x^{k}} .
$$

Furthermore, upon bounding $\left(h_{x}^{k}\right)^{-1}\left|\delta \theta_{\lambda}^{k+1}-\delta \theta_{\lambda}^{k}\right|=2\left(h_{x}^{k}\right)^{-1}\left|\Delta_{k+1}-\Delta_{k}\right|$ using (4.11), (4.3), and (4.5)-(4.6) together with similar estimates for $u_{\lambda}-\tilde{u}_{\lambda}$, we conclude that

$$
\left|\theta_{\lambda}^{\prime}\left(x^{k+1 / 2}\right)-\tilde{\theta}_{\lambda}^{\prime}\left(x^{k+1 / 2}\right)\right| \leq C h^{2}|\lambda|^{6-m} e^{-\beta|\lambda| x^{k}} .
$$

Proceeding as above, we get a bound similar to (4.9), so the proof in the nondegenerate cases is complete.

In the remaining case, $a=c=0 \underline{\zeta}_{\lambda, j}$ is a polynomial of order one in maximum, so the approximation error vanishes.

We note finally that when $\left(u_{\lambda}, v_{\lambda}\right) \sim x e^{-\mu x}$ the analysis is similar, only in this case (4.3) must be considered as an approximation to the entire system (4.4) and the analysis must be performed for vector-valued functions. 
Lemma 4.2. Assume that $\lambda \neq 0$ and that the parameters satisfy one of the following:

$$
\left\{\begin{array}{l}
b=0, a \neq 0, c \neq 0, \\
b=0, a=0, c \neq 0 .
\end{array}\right.
$$

Then for every $\underline{\vartheta}_{\lambda}=\varphi_{\lambda}(y) \underline{\zeta}_{\lambda}(x)$, such that $|\lambda| h \leq \hat{c}, \hat{c}$ small enough, there exists $\underline{\tilde{\vartheta}}_{\lambda} \in \mathcal{U}_{0, h}$ such that

$$
\|\left|\underline{\vartheta}_{\lambda}-\underline{\tilde{\vartheta}}_{\lambda}\right|||_{h} \leq\left(C_{1} h+C_{2} \lambda^{2} h^{2}\right)\left|\underline{\vartheta}_{\lambda}\right|_{2}
$$

with $C_{2}=0$ in the case $a=b=0$.

Proof. For the first case with $b=0, a \neq 0, c \neq 0$ we note that (4.1) reduces to

$$
U^{k+1}-U^{k}=i \frac{a}{4 c} \tau_{k}\left(U^{k+1}+U^{k}\right)
$$

thus providing an approximation as accurate as in the hyperbolic case in Lemma 4.1. In the second case, $a=b=0, \underline{\zeta}_{\lambda}$ is a constant, so that the approximation error vanishes again.

Lemma 4.3. Assume that $\lambda=0$ and one of the following holds:

$$
\left\{\begin{array}{l}
b=0, a \neq 0, c \neq 0, \\
b=0, a=0, c \neq 0, \\
b=0, c=0, a \neq 0 .
\end{array}\right.
$$

Then for every $\underline{\phi}_{0} \in \mathcal{U}_{0}$, there exists $\underline{\phi}_{0} \in \mathcal{U}_{0, h}$ such that

$$
\|\left.\left|\underline{\phi}_{0}-\underline{\tilde{\phi}}_{0}\right|\right|_{h} \leq\left. C h \underline{\phi}_{0}\right|_{2}
$$

Proof. These modes, having all in common that they are built on an arbitrary function $\xi \in H^{3}(0, L)$, can be treated by choosing first a suitable interpolant for $\theta_{\lambda}$ and then integrating the other components from this, paralleling the construction in [9] and leading to the optimal error bound.

In order to compute our final approximation, we need some orthogonality results.

Lemma 4.4. For $\lambda \in \Lambda$ let $\underline{\Upsilon}_{\lambda}(x, y)=\varphi_{\lambda}(y) \underline{\chi}(x), \underline{\tilde{\Upsilon}}_{\lambda}(x, y)=\tilde{\varphi}_{\lambda}(y) \underline{\tilde{\chi}}(x)$ for some $\underline{\chi}, \underline{\tilde{\chi}} \in\left[H^{1}(0, L)\right]^{5}$ and let $\varphi_{\lambda}, \tilde{\varphi}_{\lambda}$ be as in Lemmas 4.1 [4.3. Then if $\lambda, \bar{\mu} \in \Lambda, \lambda \neq \mu$, one has

$$
\mathcal{A}_{b}\left(\underline{\Upsilon}_{\lambda}, \underline{\Upsilon}_{\mu}\right)=\mathcal{A}_{m}\left(\underline{\Upsilon}_{\lambda}, \underline{\Upsilon}_{\mu}\right)=0
$$

If, in addition, $|\lambda| h_{y}<\pi,|\mu| h_{y}<\pi$, then

$$
\begin{aligned}
& \mathcal{A}_{b}\left(\underline{\Upsilon}_{\lambda}, \underline{\tilde{\Upsilon}}_{\mu}\right)=\mathcal{A}_{b}\left(\underline{\tilde{\Upsilon}}_{\lambda}, \underline{\tilde{\Upsilon}}_{\mu}\right)=0, \\
& \mathcal{A}_{m}\left(\underline{\Upsilon}_{\lambda}, \tilde{\Upsilon}_{\mu}\right)=\mathcal{A}_{m}\left(\underline{\tilde{\Upsilon}}_{\lambda}, \tilde{\Upsilon}_{\mu}\right)=0,
\end{aligned}
$$

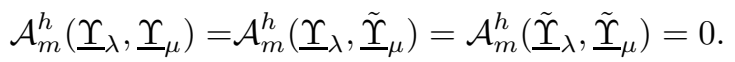

Proof. The first assertion follows from $\varphi_{\lambda}^{\prime}=i \lambda \varphi_{\lambda}$ and from the orthogonality $\left(\varphi_{\lambda}, \varphi_{\mu}\right) \doteqdot \int_{0}^{H} \varphi_{\lambda} \bar{\varphi}_{\mu} d y=0, \lambda, \mu \in \Lambda, \lambda \neq \mu$. To prove the orthogonality for 
the remaining seven bilinear expressions, we note that if the integration in the seven expressions is restricted to the domain

$$
\Omega_{n}=\left\{(x, y) \mid 0<x<L, y^{n}<y<y^{n+1}\right\},
$$

the result takes the form $e^{i(\lambda-\mu) n h_{y}} C\left(\mu, \lambda, h_{y}\right)$ in each case. The assertion then follows by summing over $n$.

Proof of Theorem [3.1. Consider first the case, when $b \neq 0$. Then it suffices to show that for each $\underline{u}=\underline{u}^{j} \in \mathcal{U}_{0}$ of the form $\underline{u}^{j}=\sum_{\lambda \in \Lambda} A_{\lambda} \varphi_{\lambda}(y) \underline{\zeta}_{\lambda, j}(x), j=1,2$, there exists $\underline{\tilde{u}}^{j} \in \mathcal{U}_{0, h}$ such that

$$
||\left|\underline{u}^{j}-\underline{\tilde{u}}^{j}\right|||_{h} \leq C_{1} h\left|\underline{u}^{j}\right|_{2}+C_{2} h^{\frac{2}{3}(s+1)}\left|\underline{u}^{j}\right|_{2+s},
$$

where $C_{2}=0$ in the elliptic and in the degenerate parabolic and hyperbolic cases. To this end, drop again the index $j$, write $\underline{\vartheta}_{\lambda}=\varphi_{\lambda}(y) \underline{\zeta}_{\lambda}(x)$, and set $\underline{\tilde{u}}=\sum_{|\lambda| \leq \lambda_{0}} A_{\lambda} \underline{\tilde{\vartheta}}_{\lambda}$, where $\underline{\tilde{\vartheta}}_{\lambda}$ is as in Lemma 4.1. Then Lemma 4.4 gives

$$
\begin{aligned}
\| \underline{u}-\underline{\tilde{u}}||_{h}^{2} & =\|\left.\left|\sum_{|\lambda| \leq \lambda_{0}} A_{\lambda}\left(\underline{\vartheta}_{\lambda}-\underline{\tilde{\vartheta}}_{\lambda}\right)+\sum_{|\lambda|>\lambda_{0}} A_{\lambda} \underline{\vartheta}_{\lambda}\right|\right|_{h} ^{2} \\
& \leq 2 \sum_{|\lambda| \leq \lambda_{0}}\left|A_{\lambda}\right|^{2}\left|\left\|\underline{\vartheta}_{\lambda}-\underline{\tilde{\vartheta}}_{\lambda}||_{h}^{2}+2 \sum_{|\lambda|>\lambda_{0}}|| A_{\lambda} \underline{\vartheta}_{\lambda}\right\|\right|_{h}^{2} .
\end{aligned}
$$

In the elliptic case and in the degenerate hyperbolic and parabolic cases we can set $\lambda_{0}=\frac{\hat{c}}{h}, \hat{c}$ sufficiently small, to obtain

$$
|||\underline{u}-\underline{\tilde{u}}| \|_{h}^{2} \leq C h^{2} \sum_{|\lambda| \leq \frac{c}{h}}\left|A_{\lambda}\right|^{2}\left|\underline{\vartheta}_{\lambda}\right|_{2}^{2}+C h^{2} \sum_{|\lambda|>\frac{c}{h}}|\lambda|^{2}\left|A_{\lambda}\right|^{2}\left|\underline{\vartheta}_{\lambda}\right|_{1}^{2} \leq C h^{2}|\underline{u}|_{2}^{2},
$$

where we used Lemma 4.1, the bound ||$\left|\underline{\vartheta}_{\lambda}\right|||_{h}^{2} \leq C\left|\underline{\vartheta}_{\lambda}\right|_{1}^{2}$, and the fact that

$$
\sum_{\lambda \in \Lambda}|\lambda|^{2 p}\left|A_{\lambda}\right|^{2}\left|\underline{\vartheta}_{\lambda}\right|_{\ell}^{2} \leq C|\underline{u}|_{\ell+p}^{2}, \quad p \geq 0 .
$$

In the nondegenerate hyperbolic and parabolic cases we get first by a similar argument that

$$
\begin{aligned}
\| \underline{u}-\left.\underline{\tilde{u}}||\right|_{h} ^{2} & \leq C h^{2}|\underline{u}|_{2}^{2}+C h^{4} \sum_{|\lambda| \leq \lambda_{0}}\left|A_{\lambda} \lambda^{p}\right|^{2}|\lambda|^{4-2 p}\left|\underline{\vartheta}_{\lambda}\right|_{2}^{2}+\left.2 \sum_{|\lambda|>\lambda_{0}} \lambda^{-2 r}|| \lambda^{r} A_{\lambda} \underline{\vartheta}_{\lambda}||\right|_{h} ^{2} \\
& \leq C\left(h^{2}|\underline{u}|_{2}^{2}+h^{4} \lambda_{0}^{2(2-p)}|\underline{u}|_{2+p}^{2}+\lambda_{0}^{-2 r}|\underline{u}|_{1+r}^{2}\right) .
\end{aligned}
$$

Balancing the last two error terms here, choosing $r=1+p, \lambda_{0} \sim h^{-\frac{2}{3}}$, we get

$$
\begin{aligned}
\|\underline{u}-\underline{\tilde{u}} \mid\|_{h}^{2} & \leq C h^{2}|\underline{u}|_{2}^{2}+C\left(h^{4} \lambda_{0}^{2(2-p)}+\lambda_{0}^{-2(1+p)}\right)|\underline{u}|_{2+p}^{2} \\
& \leq C h^{2}|\underline{u}|_{2}^{2}+C h^{\frac{4}{3}(p+1)}|\underline{u}|_{2+p}^{2}, \quad 0 \leq p \leq 1 .
\end{aligned}
$$

For the cases $b=0$, we write $\underline{u}=\varphi_{0} \underline{\phi}_{0}+\sum_{\lambda \in \Lambda \backslash\{0\}} A_{\lambda} \underline{\vartheta}_{\lambda}$ and $\underline{\tilde{u}}=\tilde{\varphi}_{0} \underline{\tilde{\phi}}_{0}+$ $\sum_{0<|\lambda| \leq \lambda_{0}} A_{\lambda} \underline{\tilde{q}}_{\lambda}$ and apply the results given in Lemmas 4.24 .4 as above. This proves the theorem together with (4.12), (4.13).

Remark 4.1. Choosing $\underline{u}=\underline{\vartheta}_{\lambda_{0}}$ in the nondegenerate hyperbolic or parabolic case so that $C_{2} \neq 0$ in Lemma 4.1 we conclude from the proof of Lemma 4.1 that

$$
\left\|\underline{u}-\left.\underline{\tilde{u}}\left|\|_{h}^{2} \sim h^{2}\right| \underline{u}\right|_{2} ^{2}+h^{\frac{4}{3}(s+1)}|\underline{u}|_{2+s}^{2}, \quad 0 \leq s \leq 1 .\right.
$$


Since obviously $\underline{\tilde{u}}$ is essentially the best approximation of $\underline{u}$, we conclude that the error bound of Theorem 3.1 is not improvable.

Remark 4.2. In the degenerate parabolic and hyperbolic shell geometries, the Fourier transform technique is actually not needed for proving Theorem 3.1 This is because in those cases the general inextensional displacement field takes the simple form

$$
\underline{u}(x, y)= \begin{cases}x \underline{\Phi}_{1}(y)+\underline{\Phi}_{2}(y) & \text { when } a=c=0, \\ y \underline{\Phi}_{1}(x)+\underline{\Phi}_{2}(x) & \text { when } b=c=0, \\ \underline{\Phi}_{1}(x)+\underline{\Phi}_{2}(y) & \text { when } a=b=0,\end{cases}
$$

which allows the 1D construction technique of [9] (cf. also [7]) when approximating $\underline{u}$. In this way one can show that Theorem 3.1 holds on a general rectangular mesh in the degenerate geometries. Also more general than semiperiodic boundary conditions can be handled in these cases (cf. 9]).

Proof of Theorem 3.2. To proceed with the case when $\underline{u}$ is not inextensional, we use again the Fourier decomposition and look for interpolants $\underline{\tilde{\vartheta}}_{\lambda}(x)$ such that $\tilde{\beta}_{i j}\left(\underline{\tilde{\vartheta}}_{\lambda}\right)=\tilde{\beta}_{i j}\left(\underline{\vartheta}_{\lambda}\right)$ and $\tilde{\rho}_{i}\left(\underline{\tilde{\vartheta}}_{\lambda}\right)=\tilde{\rho}_{i}\left(\underline{\vartheta}_{\lambda}\right)$ for $|\lambda| h \leq c, c>0$ sufficiently small, so that $\mathcal{A}_{m}^{h}\left(\underline{\vartheta}_{\lambda}-\underline{\tilde{\vartheta}}_{\lambda}, \underline{\vartheta}_{\lambda}-\underline{\tilde{\vartheta}}_{\lambda}\right)=0$ for such $\lambda$. In this case (4.1) becomes a system on inhomogeneous difference equations. In the same way as in the inextensional case, we find that when $b \neq 0$ the reduced system corresponding to (4.3) is

$$
\left(\begin{array}{l}
V \\
U
\end{array}\right)_{k+1}-\left(\begin{array}{l}
V \\
U
\end{array}\right)_{k}=\frac{1}{2} \tau_{k} M\left[\left(\begin{array}{l}
V \\
U
\end{array}\right)_{k+1}+\left(\begin{array}{l}
V \\
U
\end{array}\right)_{k}\right]+h_{x}^{k} \tilde{F}^{k},
$$

where $\tilde{F}^{k}$ is a second-order accurate approximation to $F\left(x^{k+1 / 2}\right)$ in the system

$$
\left(\begin{array}{l}
v_{\lambda} \\
u_{\lambda}
\end{array}\right)^{\prime}=\lambda M\left(\begin{array}{l}
v_{\lambda} \\
u_{\lambda}
\end{array}\right)+F=\lambda M\left(\begin{array}{l}
v_{\lambda} \\
u_{\lambda}
\end{array}\right)+\left(\begin{array}{l}
F_{1} \\
F_{2}
\end{array}\right)
$$

as satisfied by $v_{\lambda}$ and $u_{\lambda}$ with

$$
F_{1}=2 f_{12}-2 c b^{-1} f_{22}, \quad F_{2}=f_{11}-a b^{-1} f_{22},
$$

where $f_{j l}=e^{-i \lambda y} \beta_{j l}\left(\underline{\vartheta}_{\lambda}\right)$. For the error $\delta F_{j}^{k}=F_{j}\left(x^{k+1 / 2}\right)-\tilde{F}_{j}^{k}$, one gets the bounds

$$
\begin{aligned}
\left|\delta F_{j}^{k}\right| \leq & C\left(h_{x}^{2}\left\|F_{j}^{\prime \prime}\right\|_{\infty}+\lambda^{2} h_{y}^{2}\left\|F_{j}\right\|_{\infty}\right), \\
\left|\delta F_{j}^{k+1}-\delta F_{j}^{k}\right| \leq & C\left|h_{x}^{k+1}-h_{x}^{k}\right| h_{x}\left\|F_{j}^{\prime \prime}\right\|_{\infty} \\
& +C\left(h_{x}^{k+1}+h_{x}^{k}\right)\left(h_{x}^{2}\left\|F_{j}^{\prime \prime \prime}\right\|_{\infty}+\lambda^{2} h_{y}^{2}\left\|F_{j}^{\prime}\right\|_{\infty}\right),
\end{aligned}
$$

where $\|\cdot\|_{\infty}=\|\cdot\|_{L^{\infty}(0, L)}$. Assuming $|\lambda| h \leq \hat{c}$, we can again evaluate the error of (4.15), thus obtaining (we omit the details) a bound

$$
\left|\delta v_{\lambda}^{k}\right|+\left|\delta u_{\lambda}^{k}\right| \leq G_{s} h^{s}, \quad s=1,2,
$$

where

$$
G_{s} \leq C|\lambda|^{s+2}\left(\left\|v_{\lambda}\right\|_{\infty}+\left\|u_{\lambda}\right\|_{\infty}\right)+C \sum_{m=0}^{s}|\lambda|^{s-m} \sum_{j, l}\left\|f_{j l}^{(m)}\right\|_{\infty}
$$


We note that again in this case

$$
\left.\left\|\underline{\underline{\vartheta}}_{\lambda}-\underline{\tilde{\vartheta}}_{\lambda}\right\|\right|_{h} ^{2}=\mathcal{A}_{b}\left(\underline{\vartheta}_{\lambda}-\underline{\tilde{\vartheta}}_{\lambda}, \underline{\vartheta}_{\lambda}-\underline{\tilde{\vartheta}}_{\lambda}\right) .
$$

Using the (now inhomogeneous) difference equations to provide an approximation to $\psi_{\lambda}$ and its derivative, we are led to the bound

$$
\left|\varphi_{\lambda} \psi_{\lambda}-\tilde{\varphi}_{\lambda} \tilde{\psi}_{\lambda}\right|_{1} \leq G_{\psi, \lambda} h,
$$

where $G_{\psi, \lambda}$ is a constant bounded by

$$
\begin{aligned}
G_{\psi, \lambda} \leq & C \lambda^{4}\left(\left\|v_{\lambda}\right\|_{\infty}+\left\|u_{\lambda}\right\|_{\infty}\right)+C \sum_{m=0}^{3}|\lambda|^{3-m} \sum_{j, l}\left\|f_{j l}^{(m)}\right\|_{\infty} \\
& +C \sum_{m=0}^{2}|\lambda|^{2-m}\left(\left\|g_{2}^{(m)}\right\|_{\infty}+\left\|\psi^{(m)}\right\|_{\infty}\right),
\end{aligned}
$$

where $g_{2}(x)=e^{-i \lambda y} \rho_{2}\left(\underline{\vartheta}_{\lambda}\right)$.

Bounding $\left|\varphi_{\lambda} \theta_{\lambda}-\tilde{\varphi}_{\lambda} \tilde{\theta}_{\lambda}\right|_{1}^{2}$ is again a little trickier. Similar to the homogeneous case we have that

$$
\left(\begin{array}{c}
\tilde{v}_{\lambda} \\
\tilde{u}_{\lambda}
\end{array}\right)^{\prime}\left(x^{k+1 / 2}\right)=\frac{1}{h_{y}} \tan \left(\frac{1}{2} \lambda h_{y}\right) M\left[\left(\begin{array}{c}
\tilde{v}_{\lambda} \\
\tilde{u}_{\lambda}
\end{array}\right)\left(x^{k}\right)+\left(\begin{array}{c}
\tilde{v}_{\lambda} \\
\tilde{u}_{\lambda}
\end{array}\right)\left(x^{k+1}\right)\right]+\tilde{F}^{k} .
$$

The error $\delta \theta_{\lambda}^{k}$ satisfies again a similar equation to (4.10), where now $\omega_{k}$ contains additional terms due to inhomogeneity. To bound the contribution from these terms to the telescoping sum (4.10), we need the second estimate in (4.16) and a similar bound for $\delta g_{1}^{k}=g_{1}\left(x^{k+1 / 2}\right)-\tilde{g}_{1}^{k}$ where $g_{1}(x)=e^{-i \lambda y} \rho_{1}\left(\underline{\vartheta}_{\lambda}\right)$. Invoking the mesh hypothesis (B) we obtain

$$
\left|\delta \theta_{\lambda}^{k}\right| \leq \tilde{G}_{\theta, \lambda} h^{2}
$$

where $\tilde{G}_{\theta, \lambda}$ is bounded by

$$
\begin{aligned}
\tilde{G}_{\theta, \lambda} \leq & C|\lambda|^{5}\left(\left\|v_{\lambda}\right\|_{\infty}+\left\|u_{\lambda}\right\|_{\infty}\right)+C \sum_{m=0}^{4}|\lambda|^{4-m} \sum_{j, l}\left\|f_{j l}^{(m)}\right\|_{\infty} \\
& +C \sum_{m=0}^{3}|\lambda|^{2-m}\left\|g_{1}^{(m)}\right\|_{\infty} .
\end{aligned}
$$

This provides a bound for $\left|\theta_{\lambda}\left(x^{k}\right)-\tilde{\theta}_{\lambda}\left(x^{k}\right)\right|$. To bound also $\left|\theta_{\lambda}^{\prime}\left(x^{k+1 / 2}\right)-\tilde{\theta}_{\lambda}^{\prime}\left(x^{k+1 / 2}\right)\right|$, we use the mesh hypothesis (A) and apply an inverse inequality, so as to obtain the desired bound

$$
\left|\varphi_{\lambda} \theta_{\lambda}-\tilde{\varphi}_{\lambda} \tilde{\theta}_{\lambda}\right|_{1} \leq G_{\theta, \lambda} h
$$

with

$$
G_{\theta, \lambda} \leq C\left(\tilde{G}_{\theta, \lambda}+\sum_{m=0}^{2}|\lambda|^{2-m}\left\|\theta^{(m)}\right\|_{\infty}\right) .
$$

Combining finally (4.17), (4.18) we have

$$
\left.\left\|\underline{\vartheta}_{\lambda}-\underline{\tilde{\vartheta}}_{\lambda}\right\|\right|_{h} ^{2} \leq C\left(G_{\psi, \lambda}^{2}+G_{\theta, \lambda}^{2}\right) h^{2} .
$$


To finalize the analysis, we assume that $\underline{u}=\sum_{\lambda \in \Lambda} A_{\lambda} \underline{\vartheta}_{\lambda}$ and let our approximation be $\underline{\tilde{u}}=\sum_{|\lambda| \leq \lambda_{0}} A_{\lambda} \underline{\tilde{\vartheta}}_{\lambda}$. Then we have again that

$$
\begin{aligned}
\|\underline{u}-\underline{\tilde{u}} \mid\|_{h}^{2} & \leq 2 \sum_{|\lambda| \leq \lambda_{0}}\left|A_{\lambda}\right|^{2}||\left|\underline{\vartheta}_{\lambda}-\underline{\tilde{\vartheta}}_{\lambda}\right|\left\|_{h}^{2}+2 \sum_{|\lambda|>\lambda_{0}}\left|A_{\lambda}\right|^{2}|| \underline{\vartheta}_{\lambda} \mid\right\|_{h}^{2} \\
& \leq C_{1}(\underline{u}) h^{2}+\lambda_{0}^{-2 s} \sum_{|\lambda|>\lambda_{0}}||\left|\lambda^{s} A_{\lambda} \underline{\vartheta}_{\lambda}\right| \|_{h}^{2} \\
& \leq C_{1}(\underline{u}) h^{2}+C_{2}(\underline{u}) \lambda_{0}^{-2 s} t^{-2},
\end{aligned}
$$

whereby setting $\lambda_{0} h=\hat{c}$ gives

$$
\|\underline{u}-\underline{\tilde{u}} \mid\|_{h}^{2} \leq C_{1}(\underline{u}) h^{2}+C_{2}(\underline{u}) t^{-2} h^{2 s} .
$$

Here we may bound $C_{1}(\underline{u})$ from

$$
C_{1}(\underline{u}) \leq C \sum_{\lambda \in \Lambda}\left|A_{\lambda}\right|^{2}\left(G_{\psi, \lambda}^{2}+G_{\theta, \lambda}^{2}\right)
$$

using

$$
\begin{aligned}
\sum_{\lambda \in \Lambda}|\lambda|^{2 s}\left\|f_{\lambda}\right\|_{L^{\infty}(0, L)}^{2} & \leq C \sum_{\lambda \in \Lambda}|\lambda|^{2 s}\left\|f_{\lambda}\right\|_{H^{1}(0, L)}^{2} \\
& \leq C|| f \|_{s+1}^{2}, \quad f=\sum_{\lambda \in \Lambda} \varphi_{\lambda}(y) f_{\lambda}(x)
\end{aligned}
$$

as

$$
C_{1}(\underline{u}) \leq C\left(\|u\|_{6}^{2}+\|v\|_{6}^{2}+\|w\|_{5}^{2}+\|\theta\|_{3}^{2}+\|\psi\|_{3}^{2}\right) \leq C\|\underline{u}\|_{6}^{2} .
$$

Constant $C_{2}(\underline{u})$ may be bounded, using the fact that the reduction operators $\Pi_{h}^{x}$, $\Pi_{h}^{y}$ are bounded as maps from $H^{1}(\Omega)$ to $L^{2}(\Omega)$, as

$$
C_{2}(s, \underline{u}) \leq \sum_{\lambda \in \Lambda}|\lambda|^{2 s}\left(\left\|u_{\lambda}\right\|_{2}^{2}+\left\|v_{\lambda}\right\|_{2}^{2}+\left\|w_{\lambda}\right\|_{2}^{2}+\left\|\theta_{\lambda}\right\|_{1}^{2}+\|\psi\|_{1}^{2}\right) \leq C\|\underline{u}\|_{2+s}^{2} .
$$

This completes the proof for the case $b \neq 0$. When $b=0$, the analysis is similar and the final result takes the same form. We omit the details.

Remark 4.3. That the additional mesh hypothesis (B) in the proof of Theorem 3.2 is necessary, is seen by considering a single mode where $u_{\lambda}=v_{\lambda}=0, w_{\lambda}(x)=x^{3}$, $\theta_{\lambda}=w_{\lambda}^{\prime}, \psi_{\lambda}=i \lambda w_{\lambda}$, and a mesh where $h_{x}^{k}=h_{x}$ for $k$ odd and $h_{x}^{k}=h_{x} / 2$ for $k$ even. Then the above construction enforces $\tilde{u}_{\lambda}=\tilde{v}_{\lambda}=0, \tilde{w}_{\lambda}\left(x^{k}\right)=w_{\lambda}\left(x^{k}\right)$, so (4.10) holds with $\omega_{k}=\frac{1}{2}\left(h_{x}^{k}\right)^{2}$, and we conclude that

$$
\left|\theta_{\lambda}^{\prime}\left(x^{k+1 / 2}\right)-\tilde{\theta}_{\lambda}^{\prime}\left(x^{k+1 / 2}\right)\right|=c_{k}\left|2 \delta \theta_{\lambda}^{0} / h_{x}+\frac{3}{4} k h_{x}\right|+O\left(h_{x}\right),
$$

where $c_{k}=1$ for $k$ odd and $c_{k}=2$ for $k$ even. Then $\left|\varphi_{\lambda} \theta_{\lambda}-\tilde{\varphi}_{\lambda} \tilde{\theta}_{\lambda}\right|_{1} \nrightarrow 0$ as $h \rightarrow 0$.

Remark 4.4. The smoothness requirements in the proof of Theorem 3.2 are sufficient for all geometries. A slight improvement in terms of $\lambda$ is obtainable in the case of an elliptic or degenerate parabolic and/or hyperbolic shells. Moreover, in the bounds for $G_{s}, G_{\psi}, G_{\theta}$ above, somewhat less regularity is actually needed for some of the $f_{j l}$ :s. 
Remark 4.5. Option (O1) for defining $\Pi_{h}^{x}, \Pi_{h}^{y}$ fails in Theorem 3.2 because in that case we could not enforce the constraints $\mathcal{A}_{m}^{h}\left(\underline{\vartheta}_{\lambda}-\underline{\tilde{\vartheta}}_{\lambda}, \underline{\vartheta}_{\lambda}-\underline{\tilde{\vartheta}}_{\lambda}\right)=0$ for $|\lambda| h \leq c$. Indeed, $\Pi_{h}^{y}\left(\beta_{22}(\underline{u})-\beta_{22}(\underline{\tilde{u}})\right)=0$ would require that $\beta_{22}(\underline{u})$ varies linearly in $x$, otherwise $\Pi_{h}^{y} \beta_{22}(\underline{u})$ could be discontinuous at points $x^{k}$, unlike $\Pi_{h}^{y} \beta_{22}(\underline{\tilde{u}})$. This shows that when considering more general than (nearly) inextensional deformations, the error indicator (3.4) becomes very sensitive not only to the smoothness of $\underline{u}$ but also to the way the modified energy norm $\||\cdot|\|_{h}$ is extended beyond $\mathcal{U}_{h}$.

Acknowledgment. The authors wish to thank the anonymous reviewer for several valuable comments.

\section{Appendix A. Error analysis for an A-stable difference scheme}

Suppose that we are trying to solve the linear, homogeneous scalar ODE

$$
\frac{d y}{d x}=-\mu y, \quad y(0)=y^{0}
$$

for $\mu>0$ with an implicit difference scheme

$$
\tilde{y}\left(x^{k}\right)-\tilde{y}\left(x^{k-1}\right)=-\frac{1}{2} \mu \tau_{k}\left(\tilde{y}\left(x^{k}\right)+\tilde{y}\left(x^{k-1}\right)\right), \quad \tilde{y}(0)=y^{0},
$$

leading to

$$
\tilde{y}\left(x^{k}\right)=\frac{1-\frac{1}{2} \mu \tau_{k}}{1+\frac{1}{2} \mu \tau_{k}} \tilde{y}\left(x^{k-1}\right)=\sigma_{k-1} \tilde{y}\left(x^{k-1}\right) .
$$

Assume further that the $\sigma_{k}$ 's satisfy

$$
\sigma_{k}=e^{-\mu h_{x}^{k}}+O\left(h_{x}^{k} h^{n}\right),
$$

where $h_{x}^{k}=x^{k+1}-x^{k}, h=\max _{k} h_{x}^{k}, n \in \mathbb{N}$ and that the damping property holds

$$
\left|\sigma_{k}\right| \leq e^{-\beta h_{x}^{k}}
$$

with $\beta>0$. Then we can expand $y\left(x^{k}\right)-\tilde{y}\left(x^{k}\right)=e^{-\mu x^{k}} y^{0}-\sigma_{k-1} \cdots \sigma_{0} y^{0}$ as a telescoping sum, so that

$$
\begin{aligned}
\left|y\left(x^{k}\right)-\tilde{y}\left(x^{k}\right)\right| & \leq\left|\sum_{j=1}^{k}\left(\sigma_{k-1} \cdots \sigma_{j} e^{-\mu x^{j}} y^{0}-\sigma_{k-1} \cdots \sigma_{j-1} e^{-\mu x^{j-1}} y^{0}\right)\right| \\
& \leq C \sum_{j=1}^{k}\left|\sigma_{k-1} \cdots \sigma_{j} e^{-\mu x^{j-1}}\left(e^{-\mu h_{x}^{j-1}}-\sigma_{j-1}\right)\right| \\
& \leq C h^{n} \sum_{j=1}^{k}\left|\sigma_{k-1} \cdots \sigma_{j} e^{-\mu x^{j}} h_{x}^{j-1}\right| \\
& \leq C h^{n} \sum_{j=1}^{k} e^{-\beta\left(x^{k}-x^{j}\right)} e^{-\mu x^{j}} h_{x}^{j-1} \\
& \leq C h^{n} \int_{0}^{x^{k}} e^{-\beta\left(x^{k}-t\right)} e^{-\mu t} d t
\end{aligned}
$$

by (A.1), A.2). A generalization of the above reasoning to the vector case is straightforward. 


\section{REFERENCES}

1. K.J. Bathe, E.N. Dvorkin, A formulation of general shell elements - the use of mixed interpolation of tensorial components, Int. J. Numer. Methods Engrg. 22 (1986) 697-722.

2. D. Chapelle, K.J. Bathe, Fundamental considerations for the finite element analysis of shell structures, Computers \& Structures, 66 (1998) 19-36.

3. V. Havu, J. Pitkäranta, An analysis of finite element locking in a parameter dependent model problem, Helsinki University of Technology Institute of Mathematics Research Reports A420 (1999); and Internat. J. Numer. Methods Engrg. 48 (2000) 1637-1671. MR 2001c:74084

4. R.H. MacNeal, Finite Elements: Their Design and Performance (Marcel Dekker, 1994).

5. M. Malinen, J. Pitkäranta, A benchmark study of reduced-strain shell finite elements: quadratic schemes, Helsinki University of Technology Laboratory for Mechanics of Materials Research Reports TKK-Lo-26 (1999); and Internat. J. Numer. Methods Engrg. 48 (2000) 1637-1671. MR 2001c:74084

6. M. Malinen, On geometrically incompatible bilinear shell elements and classical shell models, to appear.

7. J. Pitkäranta, The first locking-free plane-elastic finite element: historia mathematica, Helsinki University of Technology Institute of Mathematics Research Reports A411 (1999); and Comput. Methods Appl. Mech. Engrg. 190 (2000), 1323-1366. CMP 2001:06

8. J. Pitkäranta, A.-M. Matache, C. Schwab, Fourier mode analysis of layers in shallow shell deformations, Research Report No. 99-18 (ETH Zürich, 1999).

9. J. Pitkäranta, The problem of membrane locking in finite element analysis of cylindrical shells, Numer. Math. 61, (1992) 523-542. MR 93b:65178

10. J. Pitkäranta, Y. Leino, O. Ovaskainen, J. Piila, Shell deformation states and the finite element method: A benchmark study of cylindrical shells, Comput. Methods Appl. Mech. Engrg. 128 (1995), 81-121. MR 96j:73085

Institute of Mathematics, Helsinki University of Technology, P.O. Box 1100, 02015 Helsinki Univ. of Tech., Finland

E-mail address: Ville.Havu@hut.fi

Institute of Mathematics, Helsinki University of Technology, P.O. Box 1100, 02015

Helsinki Univ. of Tech., Finland

E-mail address: Juhani.Pitkaranta@hut.fi 